\title{
Diagnosis of Natural Convection of Hydrocarbon Reservoirs
}

\author{
Polonsky, Dmitri \\ State Gubkin Oil \& Gas Academy, Fluid Dynamics Department \\ Leninsky prospect, 65 \\ 117917 Moscow, Russia
}

Paper presented at the 5th European Conference on the Mathematics of Oil Recovery, Leoben, Austria, 3-6 Sept. 1996

\begin{abstract}
Our goal was to study how to diagnose the convection of hydrocarbon mixture before the development of the natural reservoir. We considered this problem within the framework of convective stability analysis of equilibrium of a binary hydrocarbon mixture in the heterogeneous porous medium. The effective criteria of the stable equilibrium with respect to monotonic and oscillatory perturbations were analytically derived, and these criteria were applied to test whether the instability conditions exited in the natural gas-condensate and oil fields (Karachaganak and Tengiz, Kazakhstan). In the case of fluids having the high thermal conductivity and heat capacity, instability is feasible only in highly permeable zones. As a rule, instability is caused by oscillatory perturbations.
\end{abstract}

\section{INTRODUCTION}

Depth variation of component concentrations in gravity and heat fields of the Earth is a well-known phenomenon occurring in thick oil-gas-condensate reservoirs. The reservoirs are warmed up by the thermal field of the Earth (the characteristic value of the geothermal gradient in Europe is $3^{\circ} \mathrm{C}$ per $100 \mathrm{~m}[1])$. Generally, heating can cause the deterioration of the stationary mixture distribution resulting in convective fluxes, similar to Bénard cells observed in a heated vessel. The problem statement should account for the multicomponent nature of the reservoir fluid, because weighting of the mixture with depth has an effect opposite to the action of geothermal gradient. In addition, it is necessary to take into account the heterogeneous (anisotropic, fractured, and layered) structure of the reservoir.

Diagnosis of convection in these reservoirs is based on stability analysis of mechanical equilibrium conditions of fluid mixture before the development of the field. In this paper, we use the linear stability analysis of equilibrium. We studied the stability problem in the most general way by con- sidering the multicomponent nature of fluid to allow the kinetic Soret-Dufour effects and the contribution of gravity into energy equation. In contrast to the previous studies [2] - [9], here we take into account the multicomponent nature of the mixture, the anisotropy, and multiple-layer structure of the reservoir at a time. Exact formulas are derived for the stability criteria with respect to monotonic and oscillatory perturbations. The equilibrium state of the mixture is shown to be stable under specific conditions of natural gas-condensate reservoirs. It is the multicomponent nature of the mixture which defines the stability. Only oscillatory instabilities (which are impossible in the case of homogeneous fluids) can deteriorate the equilibrium state of a mixture possessing the high thermal conductivity and heat capacity.

\section{EQUATIONS OF FLOW IN A ANISOTROPIC POROUS LAYER}

Consider a convective flow of a binary mixture in a reservoir modeled by the system of plane horizontal porous layers with the diagonal anisotropy. In particular, some layers can be characterized by the high values of permeability. Thus, this model covers the cases of fractured media with horizontal cracks. We assume that the vertical permeability, diffusion, and heat conductivity coefficients $K_{0}^{j}, D_{0}^{j}$ and $\lambda_{0}^{j}$ differ from the horizontal ones, $K^{j}, D^{j}, \lambda^{j}$. The superscript $j=1, \ldots, r$ refers to the layer number, where numbering is from top to bottom. The flow of a binary mixture in each porous layer is described by the following system of nonlinear equations using the Boussinesq approximation $[7,8,11]$

$\operatorname{div} \mathbf{u}^{j}=0$

$m^{j} \partial_{t} C^{j}+\mathbf{u}^{j} \nabla C^{j}=\sum_{i=1}^{3} D_{i}^{j}\left(\partial_{i}^{2} C^{j}+\kappa_{T}^{j} \partial_{i}^{2} T^{j}\right) \ldots \ldots$
$-\partial_{1} p^{j}-\frac{\eta}{K^{j}} u_{1}^{j}=0, \quad-\partial_{2} p^{j}-\frac{\eta}{K^{j}} u_{2}^{j}=0 \ldots \ldots \ldots$
$-\partial_{3} p^{j}-\frac{\eta}{K_{0}^{j}} u_{3}^{j}+\rho_{0} g\left(\beta_{T}^{j} T^{j}+\beta_{C}^{j} C^{j}\right)=0 \ldots \ldots$ 
$C_{m}^{j} \partial_{t} T^{j}+C_{p} \rho_{0} \mathbf{u}^{j}\left(\nabla T^{j}+\mathbf{g} / C_{p}\right)=$

$\sum_{i=1}^{3}\left(\lambda_{i}^{j} \partial_{i}^{2} T^{j}+N^{j} \rho_{0} D_{i}^{j}\left(\partial_{i}^{2} C^{j}+\kappa_{T}^{j} \partial_{i}^{2} T^{j}\right)\right)$

where $\mathbf{u}^{j}=\left(u_{1}^{j}, u_{2}^{j}, u_{3}^{j}\right)$ is the flow velocity of the mixture through the porous layer, $m^{j}$ is the porosity, $\kappa_{T}^{j}=k_{T}^{j} / T^{j}$ is the thermodiffusion coefficient ( $k_{T}$ being the thermodiffusion ratio [13]), $C^{j}$ and $T^{j}$ are the deviations of the mass concentration of the lighter component and of the temperature from the constant values $C^{0}, T^{0}$ corresponding to an average density $\rho_{0}, p^{j}$ is the deviation of the pressure from a hydrostatic distribution corresponding to a constant value of $\rho_{0}, \beta_{T}^{j}$ and $\beta_{C}^{j}$ are the thermal and concentration expansion constants, $\eta$ is the viscosity, $C_{m}^{j}$ is the heat capacity of the saturated porous medium, $C_{p}$ is the isobaric heat capacity of the mixture, $\mathbf{g}$ is the gravity acceleration. $N^{j}$ is $\kappa_{T}^{j}\left(\partial \mu^{j} / \partial C^{j}\right)_{p, T}$, where $\mu^{j}$ is the effective chemical potential of the mixture [13]. Indices 1 and 2 correspond to the horizontal grids, and index 3 to the vertical grid. According to the above notation, $D_{1}^{j}=D_{2}^{j}=D^{j}, D_{3}^{j}=D_{0}^{j}$, and $\lambda_{1}^{j}=\lambda_{2}^{j}=\lambda^{j}$, $\lambda_{3}^{j}=\lambda_{0}^{j}$. Therefore, these parameters of density, viscosity, and isobaric heat capacity are common for all layers. Other parameters (dynamic variables and conductivity coefficients) in the equations (1) - (5) are individual for each layer.

Equations (1) and (2) express the mass conservation for the mixture as a whole (continuity equation) and for the light component (diffusion equation). Equations (3), (4) express Darcy's law (momentum equation). (5) is the equation of the heat transfer, being the consequence of the energy conservation law.

Under the conditions of Boussinesq approximation, thermal and concentration compressibility of the mixture are taken into account in the equation of motion (4)only. Assuming that the values of $p^{j}, T^{j}, C^{j}$ differ insignificantly from given constant values $p^{0}, T^{0}, C^{0}$, we consider that all coefficients in the $(1)-(5)$ are constant.

\section{LINEARIZATION IN THE NEIGHBORHOOD OF MECHANICAL EQUILIBRIUM}

The conditions of mechanical equilibrium for the above system of equations were derived by a standard technique [12]. We investigated a steady-state solution to the system (1) - (5) which describes the temperature and concentration variations with depth:

$$
T^{j}=T_{s}^{j}=T^{0}-A^{j} x_{3}, \quad C^{j}=C_{s}^{j}=C^{0}-B^{j} x_{3} .
$$

This equilibrium state of this sort occurs in a horizontal layer of porous medium bounded by planes
$x_{3}=L^{j}$ and $x_{3}=L^{j+1}$ if constant values of the temperature and concentration are set on those planes. In that case, $A^{j}=\Delta T^{j} / L^{j}, B^{j}=\Delta C^{j} / L^{j}$ where $\Delta T^{j}$ and $\Delta C^{j}$ are the temperature and concentration differences across the two planes.

It is important that the temperature and concentration gradients are connected by the continuity conditions for the heat and mass flow on the boundaries of the adjacent layers:

$\lambda_{0}^{j} A^{j}=\lambda_{0}^{j+1} A^{j+1}, \quad D_{0}^{j} B^{j}=D_{0}^{j+1} B^{j+1}$

To investigate the stability of the above equilibrium state we expand the hydrodynamic variables into the sums of equilibrium solutions and small perturbations:

$P^{j}=P_{s}^{j}+P^{\prime j}, \quad T^{j}=T_{s}^{j}+T^{\prime j}$,

$C^{j}=C_{s}^{j}+C^{\prime j}, \quad \mathbf{u}^{j}=\mathbf{u}^{\prime} j$

Substituting (8) in the system of equations (1) - (5) for Boussinesq approximation, neglecting squares of the perturbation terms, and crossing out any primes in the notation, we arrive at the following equations:

$\operatorname{div} \mathbf{u}^{j}=0$

$\partial_{t} C^{j}-B^{j} u_{3}^{j}=\sum_{i=1}^{3} D_{i}^{j}\left(\partial_{i}^{2} C^{j}+\kappa_{T}^{j} \partial_{i}^{2} T^{j}\right)$

$-\partial_{1} p^{j}-\frac{\eta}{K^{j}} u_{1}^{j}=0, \quad-\partial_{2} p^{j}-\frac{\eta}{K^{j}} u_{2}^{j}=0 \ldots \ldots$

$-\partial_{3} p^{j}-\frac{\eta}{K_{0}^{j}} u_{3}^{j}+\rho_{0} g\left(\beta_{T}^{j} T^{j}+\beta_{C}^{j} C^{j}\right)=0 \ldots \ldots$

$C_{m}^{j} \partial_{t} T^{j}-C_{p} \rho_{0}\left(A^{j}+g / C_{p}\right) u_{3}^{j}=$

$\sum_{i=1}^{3}\left(\lambda_{i}^{j} \partial_{i}^{2} T+N^{j} \rho_{0} D_{i}^{j}\left(\partial_{i}^{2} C^{j}+\kappa_{T}^{j} \partial_{i}^{2} T^{j}\right)\right) \ldots \ldots$

Rewrite the equations of the last system in dimensionless form. The characteristic length is taken to be the layer thickness $L^{j}$, characteristic time $K_{0}^{j} \rho_{0} / \eta$, velocity $\lambda_{0}^{j} / \rho_{0} C_{p} L^{j}$, temperature $A^{j} L^{j}$, pressure $\eta \lambda_{0}^{j} / \rho_{0} C_{p} K_{0}^{j}$, and concentration $B^{j} L^{j} \lambda_{0}^{j} / \rho_{0} C_{p} K_{0}^{j}$. Switching to dimensionless variables with the aid of these units, we obtain:

$\operatorname{div} \mathbf{u}^{j}=0$

$\mathrm{P}_{\mathrm{d}}^{j} \partial_{t} C^{j}-u_{3}^{j}=\Delta_{D} C^{j}+\mathrm{T}_{\mathrm{d}}^{j} \Delta_{D} T^{j}$

$-\partial_{1} p^{j}-\left(f_{k}^{j}\right)^{-1} u_{1}^{j}=0,-\partial_{2} p^{j}-\left(f_{k}^{j}\right)^{-1} u_{2}^{j}=0 \ldots$

$-\partial_{3} p^{j}-u_{3}^{j}+\left(\mathrm{R}^{j} T^{j}+\mathrm{R}_{\mathrm{d}}^{j} C^{j}\right)=0$.

$\mathrm{P}^{j} \partial_{t} T^{j}-\left(1+\mathrm{G}^{j}\right) u_{3}^{j}=$

$\left(\Delta_{l}+\mathrm{D}_{\mathrm{t}}^{j} \Delta_{D}\right) T^{j}+\left(\mathrm{D}_{\mathrm{t}}^{j} / \mathrm{T}_{\mathrm{d}}^{j}\right) \Delta_{D} C^{j}$ 
The differential operators $\Delta_{D}, \Delta_{l}$ in the equations (15), (18) are defined by

$$
\Delta_{D}=\sum_{i=1}^{3}\left(D_{i}^{j} / D_{3}^{j}\right) \partial_{i}^{2}, \quad \Delta_{l}=\sum_{i=1}^{3}\left(\lambda_{i}^{j} / \lambda_{3}^{j}\right) \partial_{i}^{2} \ldots \ldots
$$

The dimensionless parameters in the system (14) (19) are as follows:

$$
\begin{aligned}
& \mathrm{R}^{j}=\frac{\rho_{0}^{2} g \beta_{T}^{j} A^{j} L^{j 2} K_{0}^{j} C_{p}}{\eta \lambda_{0}^{j}}, \quad \mathrm{P}^{j}=\frac{C_{m}^{j} L^{j 2} \eta}{K_{0}^{j} \lambda_{0}^{j} \rho_{0}} \\
& \mathrm{R}_{\mathrm{d}}^{j}=\frac{\rho_{0} g \beta_{C}^{j} B^{j} L^{j 2} K_{0}^{j}}{\eta D_{0}^{j}}, \quad \mathrm{P}_{\mathrm{d}}^{j}=\frac{m^{j} L^{j 2} \eta}{K_{0}^{j} D_{0}^{j} \rho_{0}} \\
& \mathrm{~T}_{\mathrm{d}}^{j}=\frac{A^{j} D^{j} C_{p} \kappa_{T}^{j} \rho_{0}}{B^{j} \lambda_{0}^{j}}, \quad \mathrm{D}_{\mathbf{t}}^{j}=\frac{N^{j} D_{0}^{j} \kappa_{T}^{j} \rho_{0}}{\lambda_{0}^{j}}, \\
& f_{k}^{j}=\frac{K^{j}}{K_{0}^{j}}, \quad f_{d}^{j}=\frac{D^{j}}{D_{0}^{j}}, \quad f_{\lambda}^{j}=\frac{\lambda^{j}}{\lambda_{0}^{j}}, \quad G^{j}=\frac{g}{A^{j} C_{p}}
\end{aligned}
$$

The flow and diffusion-flow Rayleigh numbers $\mathrm{R}^{j}$ and $\mathrm{R}_{\mathrm{d}}{ }^{j}$ determine the relations between the components of the driving force associated with the presence of temperature and concentration gradients, and the corresponding dissipative mechanisms (thermal conductivity and diffusion). The relations between the conductivity (mobility) in the porous medium, $K_{0}^{j} / \eta$, and the values of the parameters, which determine the rate of dissipative processes, are expressed by the flow and diffusionflow Prandtl numbers $\mathrm{P}^{j}$ and $\mathrm{P}_{\mathrm{d}}{ }^{j}$. The parameters $\mathrm{T}_{\mathrm{d}}{ }^{j}$ and $\mathrm{D}_{\mathrm{t}}{ }^{j}$, which correspond the Soret-Dufour effects, characterize the thermal diffusion, as compared to diffusion, and diffusion thermal conductivity, as compared to ordinary thermal conductivity, respectively. The contribution of gravity in comparison to that of the temperature gradient in the layer is determined by the parameter $\mathrm{G}^{j}$. The parameters $f_{k}^{j}, f_{\lambda}^{j}, f_{d}^{j}$, similar to those introduced in [8], characterize the permeability, diffusion and the heat conductivity anisotropy in the porous medium. The last two parameters are "hidden" in (19).

We studied the system (14) - (18) under the homogeneous boundary conditions for the first $\left(x_{3}=\right.$ $\left.L^{1}\right)$ and last $\left(x_{3}=L^{r}\right)$ plane layers (all perturbations vanish at the boundaries):

$u_{3}^{j}=T^{j}=C^{j}=0,(j=1, r)$

For other layers, the perturbation of temperature, concentration, and vertical component of velocity, are related by the continuity conditions:

$$
u_{3}^{j}=T^{j}=C^{j}=u_{3}^{j+1}=T^{j+1}=C^{j+1},(j=2, r-1)
$$

\section{NORMAL PERTURBATIONS AND STABILITY DIAGRAMS}

Henceforward, unless otherwise stated, we will omit superscript " $j$ " in the notation.

The investigation of the system (14) - (18) is reduced to the standard procedure of determining the normal perturbations spectrum [12]:

$$
\begin{aligned}
& \left\{u_{i}, p, T, C\right\}= \\
& \left\{w_{i}, \zeta, \theta, \xi\right\} \exp \left(-\omega t+i\left(k_{1} x_{1}+k_{2} x_{2}\right)\right)
\end{aligned}
$$

here $\omega$ is the perturbation decrement, $k_{1}$ and $k_{2}$ are wave numbers, $w_{i}, \zeta, \theta, \xi$ are the perturbation amplitudes of the values $u_{i}, p, T, C$, respectively, the amplitudes being the functions of $x_{3}$. Due to the fact that the linear operator associated with eqs. (14) - (18) is non-hermitian, the value $\omega$ may become complex parameter, and we have generally to expect solutions with $\omega \neq 0$. The stability criterion with respect to normal perturbations has the form of $\operatorname{Re} \omega \geq 0$. Real values of $\omega$ correspond to monotonic perturbations, and complex values, to oscillatory perturbations.

By substituting the waves (22) to the (14) - (18) we arrive at the system of linear differential equations for the perturbation amplitudes $u_{i}, p, T, C$, similary to [8]. By transformations of this system, we find the simple one-parameter system of solutions satisfying the boundary conditions (20)-(21):

$w_{i}=w_{i, 0} \sin \pi n x_{3}, \quad \theta=\theta_{0} \sin \pi n x_{3}, \ldots \ldots \ldots \ldots(23)$

$\xi=\xi_{0} \sin \pi n x_{3}, \quad(n=1,2,3 \ldots) \ldots \ldots \ldots \ldots(24)$

When these solutions are substituted in (14) - (22), we obtain the system of homogeneous linear equations for $w_{i, 0}, \theta_{0}$ and $\xi_{0}$. This system has a nontrivial solution when its determinant is equal to zero:

$a \omega^{2}+b \omega+d=0$

where

$a=\mathrm{PP}_{\mathrm{d}} F_{k}$

$b=\left(F_{k}-\pi^{2} n^{2}\right)\left(\mathrm{R}_{\mathrm{d}} \mathrm{P}+(1+\mathrm{G}) \mathrm{R}_{\mathrm{d}}\right)$

$-F_{k}\left(\mathrm{P} F_{d}+\mathrm{P}_{\mathrm{d}}\left(F_{\lambda}+\mathrm{D}_{\mathrm{t}} F_{d}\right)\right)$,

$d=F_{k} F_{d} F_{\lambda}+\left(F_{k}-\pi^{2} n^{2}\right) \times\left(\mathrm{R} F_{d}\left(\mathrm{D}_{\mathrm{t}} / \mathrm{T}_{\mathrm{d}}-1-\mathrm{G}\right)\right.$

$\left.+\mathrm{R}_{\mathrm{d}}\left(\mathrm{T}_{\mathrm{d}}(1+\mathrm{G}) F_{d}-\left(\mathrm{D}_{\mathrm{t}} F_{d}+F_{\lambda}\right)\right)\right)$,

$F_{k}=\pi^{2} n^{2}+f_{k} k^{2}, \quad F_{\lambda}=\pi^{2} n^{2}+f_{\lambda} k^{2}$,

$F_{d}=\pi^{2} n^{2}+f_{d} k^{2}, \quad k^{2}=k_{1}^{2}+k_{2}^{2}$ 
The quadratic equation (25) has solutions $\omega_{1,2}$ with positive real parts if $b<0, d>0$. An equilibrium is stable if this condition is satisfied, and $b \leq 0$, $d=0$, when $\omega_{1} \geq \omega_{2}=0$. Monotonic instability $\left(\operatorname{Re} \omega_{2} \leq 0, \operatorname{Im} \omega_{2}=0\right)$ is observed in three cases: 1) if $d<0,2)$ if $d=0, b>0$, and 3) if $d>0$, $b>0$, and the discriminant $\Delta=b^{2}-4 a d$ is positive. The region of oscillatory instability corresponding to complex solutions $\omega_{1,2}$ is determined by the conditions $b>0, d>0, \Delta<0$. Purely imaginary perturbations $(\operatorname{Re} \omega=0)$ occur when $b=0$ and $d>0$. The frequency of these perturbations is equal to $(d / a)^{1 / 2}$.

Thus, in the space of dimensionless parameters $\mathcal{M}$, the region of stability and different regions of instability are separated by hypersurfaces:

$d=0$.

$b=0$.

$\Delta=b^{2}-4 a d=0$.

It is convenient to consider sections of the space $\mathcal{M}$ by planes parallel to the coordinate plane $\left(R_{d}, R\right)$. The hypersurfaces (26)-(28) are mapped on these planes by the straight lines $b=0, d=0$ and by the quadratic curve $\Delta=0$.

Since $b^{2}$ is a perfect square, and the term $4 a d$ is linear with respect to $R, R_{d}$, the curve $\Delta=0$ is parabola with its axis parallel to the straight line $b=0$. This parabola lies in the half-plane $d=b^{2} / 4 a \geq 0$. Since, for $b=0$ and $d=0$, the discriminant $\Delta=b^{2}-4 a d$ is also zero, the parabola (28) passes through the point $F$ of intersection of the straight lines (26) and (27). Moreover, since for $b=0$ we have:

$$
\frac{\partial \Delta}{\partial \mathrm{R}}=-4 a \frac{\partial d}{\partial \mathrm{R}}, \quad \frac{\partial \Delta}{\partial \mathrm{R}_{\mathrm{d}}}=-4 a \frac{\partial d}{\partial \mathrm{R}_{\mathrm{d}}}
$$

then the straight line $d=0$ is tangent to this parabola at the point $F$.

The characteristic curves $(26)-(28)$ for the condition that $\mathrm{P}_{\mathrm{d}} / \mathrm{P}>F_{d} / F_{\lambda}$ are presented on Fig.1, the other cases can be considered similarly to [7],[8]. The lines $Y H, E F$ and $V F W$ are defined by equations (26), (27) and (28), respectively. If the slope of the line $Y H$ is greater than that of the line $E F$ parallel to the axis of the parabola $V F W$, then the parabola lies under the line $Y H$ (Fig.1,a), otherwise (under condition $\mathrm{P}_{\mathrm{d}} / \mathrm{P}<F_{d} / F_{\lambda}$ ) it lies above this line. The region of absolute stability lies inside the angle $E F H$ formed by the ray $F E$ of straight line (27) and ray $F H$ of straight line (26). In the region $E F V$, instability with oscillatory perturbations is observed. The union of the lines $V F$ and $F H$ forms the lower boundary of the region of monotonic instability.

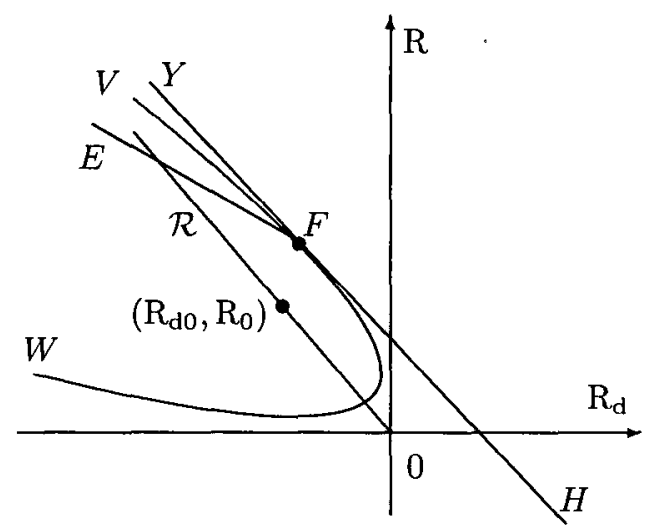

Fig. 1: STABILITY DIAGRAMS ON THE PLANE $\left(\mathrm{R}_{\mathrm{d}}, \mathrm{R}\right)$ FOR $\mathrm{P}_{\mathrm{d}} / \mathrm{P}>F_{d} / F_{\lambda}$.

The stability diagrams are easier to investigate if we neglect the gravity and cross effects: $G=T_{d}=$ $\mathrm{D}_{\mathbf{t}}=0$. In this case, we have:

$$
\begin{aligned}
& b=\left(F_{k}-\pi^{2} n^{2}\right)\left(\mathrm{R}_{\mathrm{d}} \mathrm{P}+\mathrm{RP}_{\mathrm{d}}\right)-F_{k}\left(\mathrm{P} F_{d}+\mathrm{P}_{\mathrm{d}} F_{\lambda}\right) \\
& d=F_{k} F_{d} F_{\lambda}-\left(F_{k}-\pi^{2} n^{2}\right)\left(\mathrm{R}_{\mathrm{d}} F_{\lambda}+\mathrm{R} F_{d}\right) \ldots \ldots
\end{aligned}
$$

The analysis of stability diagrams using the plane $\left(R_{d}, R\right)$ can be considered as similar to one given above for a multiple-layer system, because Rayleigh and Prandtl numbers for the adjacent layers according to $(7)$ are related by:

$$
\mathrm{R}^{j+1}=\mathrm{R}^{j} \Omega_{L} \Omega_{K}\left(\frac{\lambda_{0}^{j}}{\lambda_{0}^{j+1}}\right)^{2}
$$

$\mathrm{P}^{j+1}=\mathrm{P}^{j} \Omega_{L} \Omega_{K}^{-1} \frac{\lambda_{0}^{j} C_{m}^{j+1}}{\lambda_{0}^{j+1} C_{m}^{j}}$

$\mathrm{R}_{\mathrm{d}}^{j+1}=\mathrm{R}_{\mathrm{d}}{ }^{j} \Omega_{L} \Omega_{K}\left(\frac{D_{0}^{j}}{D_{0}^{j+1}}\right)^{2}$.

$\mathrm{P}_{\mathrm{d}}^{j+1}=\mathrm{P}_{\mathrm{d}}^{j} \Omega_{L} \Omega_{K}^{-1} \frac{D_{0}^{j} m^{j+1}}{D_{0}^{j+1} m j}$

where scale $\Omega_{L}$ and permeability factors $\Omega_{K}$ are follows

$\Omega_{L}=\left(\frac{L^{j+1}}{L^{j}}\right)^{2}, \quad \Omega_{K}=\frac{K_{0}^{j+1}}{K_{0}^{j}}$

Relations between $\mathrm{T}_{\mathrm{d}}{ }^{j}, \mathrm{D}_{\mathrm{t}}{ }^{j}, G^{j}(j=r, r+1)$ may be obtained from (7) similary. 


\section{CRITERIA OF STABILITY FOR A SEPARATE LAYER}

In the previous section, we derived the stability criteria depending on the wave numbers $n, k$. Now we will derive criteria of absolute stability, which are fulfilled for all the wave numbers.

Fig. 1 shows that the region of stability is the intersection of two half-planes, $b<0$ and $d>0$. The inequality $b<0$ can be rewritten in the quadratic form

$g(\gamma)=r_{0} \gamma^{2}+r_{1} \gamma+r_{2}<0, \gamma=k^{2}>0$.

The coefficients $r_{j}(j=0,2)$ depend on dimensionless parameters as well as on the integer wave number $n$

$$
\begin{aligned}
r_{0}= & -f_{k}\left(\mathrm{P} f_{d}+\mathrm{P}_{\mathrm{d}}\left(f_{\lambda}+\mathrm{D}_{\mathrm{t}} f_{d}\right)\right), \\
r_{1}= & -\pi^{2} n^{2}\left[\mathrm{P}\left(f_{d}+f_{k}\right)+\mathrm{P}_{\mathrm{d}}\left(f_{\lambda}+\mathrm{D}_{\mathrm{t}} f_{d}\right.\right. \\
& \left.\left.+\left(1+\mathrm{D}_{\mathrm{t}}\right) f_{k}\right)\right]+f_{k}\left(\mathrm{R}_{\mathrm{d}} \mathrm{P}+\mathrm{RP}_{\mathrm{d}}(1+\mathrm{G})\right), \\
r_{2}= & -\pi^{4} n^{4}\left(\mathrm{P}+\mathrm{P}_{\mathrm{d}}\left(1+\mathrm{D}_{\mathrm{t}}\right)\right)
\end{aligned}
$$

Similarly, the inequality $d>0$ is transformed to the form of

$$
f(\gamma)=l_{0} \gamma^{3}+l_{1} \gamma^{2}+l_{2} \gamma+l_{3}<0, \gamma=k^{2}>0 \ldots
$$

where

$$
\begin{aligned}
l_{0}= & -f_{k} f_{\lambda} f_{d}, \\
l_{1}= & -\pi^{2} n^{2}\left(f_{\lambda} f_{d}+f_{k} f_{\lambda}+f_{k} f_{d}\right) \\
& +\left[\mathrm{R}\left(1+\mathrm{G}-\mathrm{D}_{\mathrm{t}} / \mathrm{T}_{\mathrm{d}}\right)\right. \\
& \left.+\mathrm{R}_{\mathrm{d}}\left(\mathrm{D}_{\mathrm{t}}-\mathrm{T}_{\mathrm{d}}(1+\mathrm{G})\right)\right] f_{d} f_{k}+\mathrm{R}_{\mathrm{d}} f_{k} f_{\lambda}, \\
l_{2}= & -\pi^{4} n^{4}\left(f_{d}+f_{\lambda}+f_{k}\right)+\pi^{2} n^{2} f_{k} \times \\
& {\left[\left(\mathrm{R}_{\mathrm{d}}+\mathrm{R} / \mathrm{T}_{\mathrm{d}}\right)\left(\mathrm{D}_{\mathrm{t}}-\mathrm{T}_{\mathrm{d}}(1+\mathrm{G})\right)+\mathrm{R}_{\mathrm{d}}\right], } \\
l_{3}= & -\pi^{6} n^{6}
\end{aligned}
$$

Consider the inequality (37) characterizing the stability with respect to oscillatory perturbations. Note that we can set $\mathrm{n}=1$ in the coefficients $r_{i}$. After such change the left part of the inequality will only increase.

Since $r_{0}<0$ (and $r_{2}<0$ ), the inequality (37) is valid, when either the equation $g(\gamma)=0$ has no positive real solutions, or this equation has no solutions at all, which is expressed by

Either $r_{1}<0$ or $r_{1}^{2}-4 r_{0} r_{2}<0$.

In the case when $f_{k}=f_{\lambda}=f_{d}$, the inequalities (39) can be simplified. After algebraic transformations, we obtain the only condition

$R_{d} P+R P_{d}(1+G)<4 \pi^{2}\left(P+P_{d}\left(1+D_{t}\right)\right) \ldots$

Consider conditions of the polynomial negativity $f(\gamma)$ defined by $(38)$. These conditions characterize the stability with respect to monotonic perturbations.

Because $l_{0}<0$, the inequality (38) is valid if the equation $f(\gamma)=0$ has no positive solutions. The conditions for this can be simply obtained with the aid of the Cardano's transformation $\left(y=x+l_{1} / 3 l_{0}\right)$. Any of the three conditions following below is sufficient for the correctness of (38), and it follows from (38) that at least one of these conditions is valid.

1. The derivative $f^{\prime}(\gamma)$ has no solutions (and the function $f(\gamma)$ is decreasing, $f(0)$ being less than zero). This is expressed by

$$
3 l_{0} l_{2}<l_{1}^{2} \text {. }
$$

2. The derivative $f^{\prime}(\gamma)$ has real solutions, none of which is positive (and the function $f(\gamma)$ is decreasing while $\gamma>0$ ). This is valid if

$$
l_{1}<0 \text { and } l_{2}<0 \text {. }
$$

3. The derivative $f^{\prime}(\gamma)$ has a solution, $\gamma_{\max }$, in which the function $f(\gamma)$ reaches maximum. However, $f\left(\gamma_{\max }\right)<0$. This condition is expressed by the two inequalities,

$$
\begin{aligned}
& 2 l_{1}^{3}-9 l_{0} l_{1} l_{2}+27 l_{0}^{2} l_{3}<0, \quad \text { and } \\
& l_{1} l_{2}-27 l_{3}^{2} l_{0}^{3}-4 l_{1}^{3} l_{3} \\
& \quad+18 l_{0} l_{1} l_{2} l_{3}-4 l_{0} l_{2}^{3}<0 \ldots \ldots \ldots
\end{aligned}
$$

For real reservoir conditions, testing for the inequalities (42) is sufficient to prove the stability with respect to monotonic perturbations. In these inequalities, one can set $n=1$.

The conditions (41) - (43) can be simplified in the case of $f_{k}=f_{\lambda}=f_{d}$. In this case, the equation $f(\gamma)=0$ has the negative solution $-\pi^{2} n^{2} / f_{k}$. By dividing $f(\gamma)$ by $\gamma+\pi^{2} / f_{k}$ and by comparing the resulting quadratic polynomial with zero, we obtain, after simple transformations, the only condition of stability with respect to monotonic perturbations:

$\mathrm{R}_{\mathrm{d}}\left(\mathrm{D}_{\mathrm{t}}+1-\mathrm{T}_{\mathrm{d}}(1+\mathrm{G})\right)$

$+\mathrm{R}\left(1+\mathrm{G}-\mathrm{D}_{\mathrm{t}} / \mathrm{T}_{\mathrm{d}}\right)<4 \pi^{2}$

Note that the conditions (40) and (44) are the same in both isotropic and anisotropic cases (cf. $[7,11]$ ). The point is that these conditions were obtained on assumption that $f_{k}=f_{\lambda}=f_{d}$. This means that the processes of convection, diffusion, and heat conduction characteristic for a physical system under consideration are equally retarded (or accelerated) in all directions, and the stability of the system is not affected by whether or not it is anisotropic.

It should be remarked that we can rewrite the conditions (40) and (44) using relations (32)-(36). In such case, to diagnose convection in a multiplelayer it will suffice to know the temperature and concentration gradients values only for the upper layer. 


\section{CRITICAL PERMEABILITIES AND CONCENTRATION GRADIENTS}

The critical permeabilities for a separate layer are determined as threshold values above which convection is observed $[7,11]$. In the plane of the Rayleigh numbers, consider the arbitrary point $\left(R_{d 0}, R_{0}\right)$. As the permeability varies, this point describes a ray $\mathcal{R}$ with the vertex at the origin of coordinates, while the boundaries of the region of instability remain invariant (see Fig.1). The points of intersection of the ray with these boundaries give the values of the critical permeabilities $K_{m}$ and $K_{o}$ for monotonic and oscillatory instability, respectively. If the ray does not intersect a boundary of the region of instability, then the equilibrium is stable at any value of permeability. It is the case of absolute stability.

We will define the criterion of absolute stability for a binary mixture in an anisotropic porous medium. Consider typical reservoir conditions, as the following relations between dimensionless parameters take place: $R>0, R_{d}\left\langle 0,\left|R_{d}\right| \gg|R|\right.$, $\mathrm{P}_{\mathrm{d}}>\mathrm{P}$. Other cases of equilibrium are considered by analogy.

For the sake of simplicity we also neglect the gravity and cross effects: $G=T_{d}=D_{t}=0$. Then, the boundaries of monotonic and oscillatory perturbations $Y H, E F$ (Fig.1) are defined by the equations $b=0, d=0$ with $b$ and $d$ given by (30), (31).

For monotonic instability to be possible the ray $\mathcal{R}$ must intersect the boundary $Y H$. To do so, its slope must exceed absolute value of the slope of the straight line (31). This value varies from 1 to $f_{\lambda} / f_{d}$. Thus the condition that the monotonic instability is possible at some permeability is

$\phi_{m}=\frac{\mathrm{R}_{0}}{\left|\mathrm{R}_{\mathrm{d} 0}\right|}=\frac{D \rho_{0} C_{p} \beta_{T} A}{\beta_{c}|B| \lambda}>\min \left(1, f_{\lambda} / f_{d}\right) \ldots \ldots$

Similarly, the condition of possibility of the oscillatory instability has the form of

$$
\frac{\left|\mathrm{R}_{\mathrm{d} 0}\right|}{\mathrm{R}_{0}}<\frac{\mathrm{P}_{\mathrm{d}}}{\mathrm{P}}, \quad \text { or } \quad \phi_{o}=\frac{\beta_{c}|B| C_{m}}{m \rho_{0} C_{p} \beta_{T} A}<1 \ldots \ldots
$$

The critical permeability $K_{m}$ with respect to the monotonic perturbations is defined from the intersection point of the ray $\mathcal{R}$ with the boundary $d=0$ and, then, by minimizing of this value:

$K_{m}=\min _{k, n} \frac{F_{k} F_{\lambda} F_{d}}{\left(F_{k}-\pi^{2} n^{2}\right)\left(\mathrm{r}_{\mathrm{d}} F_{\lambda}+\mathrm{r} F_{d}\right)} \ldots \ldots \ldots \ldots$

where $r_{d}=R_{d} / K, r=R / K$. This expression allows an exact minimization in the case of $f_{\lambda}=f_{d}$ (and $F_{\lambda}=F_{d}$ ). In the minimum point $n=1, k^{2}=$ $\pi^{2} /\left(f_{k} f_{d}\right)^{1 / 2}$, and

$K_{m}=\frac{\pi^{2}(1+\alpha)^{2}}{\mathrm{r}_{\mathrm{d}}+\mathrm{r}}, \quad \alpha=\sqrt{f_{d} / f_{k}} \ldots$
To find the critical permeability with respect to oscillatory perturbations $K_{o}$ one must minimize by $n, k$ the value of

$K_{o}(n, k)=\frac{F_{k}}{F_{k}-\pi^{2} n^{2}} \frac{\mathrm{p} F_{d}+\mathrm{p}_{\mathrm{d}} F_{\lambda}}{\mathrm{r}_{\mathrm{d}} \mathrm{p}+\mathrm{rp}_{\mathrm{d}}}$

where $\mathrm{p}_{\mathrm{d}}=K \mathrm{P}_{\mathrm{d}}, \mathrm{p}=K \mathrm{P}$. The minimum is reached when

$n=1, \quad k^{2}=\pi^{2} \sqrt{\frac{\mathrm{p}+\mathrm{p}_{\mathrm{d}}}{f_{k}\left(\mathrm{p} f_{d}+\mathrm{p}_{\mathrm{d}} f_{\lambda}\right)}}$.

The expression for $K_{o}$ is especially simple and similar to (48) if $f_{\lambda}=f_{d}$. In this case we have

$K_{o}=\frac{\pi^{2}(1+\alpha)^{2}\left(\mathrm{p}+\mathrm{p}_{\mathrm{d}}\right)}{\mathrm{r}_{\mathrm{d}} \mathrm{p}+\mathrm{p}_{\mathrm{d}} \mathrm{r}}$

The equations (48) and (51) are the generalizations of the formula obtained in [3] for homogeneous liquid in an anisotropic porous medium. The formula [3] is in good agreement with the experiments [14].

Note that expressions (48) and (51) give values $K_{m, o}<0$ in a number of cases. This means that the intersection with the boundaries of the region of stability of the ray $\mathcal{R}$ is empty. But the origin of coordinates and a small neighborhood of this point always lie within the range of stability. Thhus, the condition $K_{m, o}<0$ is a criterion of absolute stability different from the inequalities (45), (46).

The dependencies for critical concentration gradients derived from these inequalities in the case of $f_{\lambda}=f_{d}$, are as following:

$$
\begin{aligned}
B_{m, c r} & =-\frac{D_{0}}{\lambda_{0}}\left(\frac{\rho_{0} C_{p} \beta_{T} A}{\beta_{c}}\right) \ldots \ldots \ldots \ldots \ldots \ldots \ldots \ldots \ldots \ldots \ldots \ldots \ldots \ldots \ldots
\end{aligned}
$$

Since $D_{0} C_{m} / m \lambda_{0}=10^{-3}-10^{-1}$, we obtain $\left|B_{m, c r}\right|<\left|B_{o, c r}\right|$.

A qualitative picture of the dependencies of critical permeabilities on the value $B$ of the concentration gradient is shown in Fig.2. The region of absolute stability corresponds to the value of $B$ changing from $-\infty$ to the point $M$. The section $(M N)$ defines the region where only oscillatory instabilities are possible. In the region $[N,+\infty)$, both monotonic and oscillatory instabilities are possible. The permeabilities at which the equilibrium is stable lie under the bold line.

Consider the dependencies of critical permeabilities on the concentration gradient under conditions of the two-layered system. A qualitative picture for such case is shown in Fig. 3. The points $B_{m}^{1}, B_{m}^{2}, B_{o}^{1}$, and $B_{o}^{2}$ (eqs. (7), (52)-(53)) corresponds to the values of critical gradients, where numbers of the layers are indicated by the superscripts, and the 


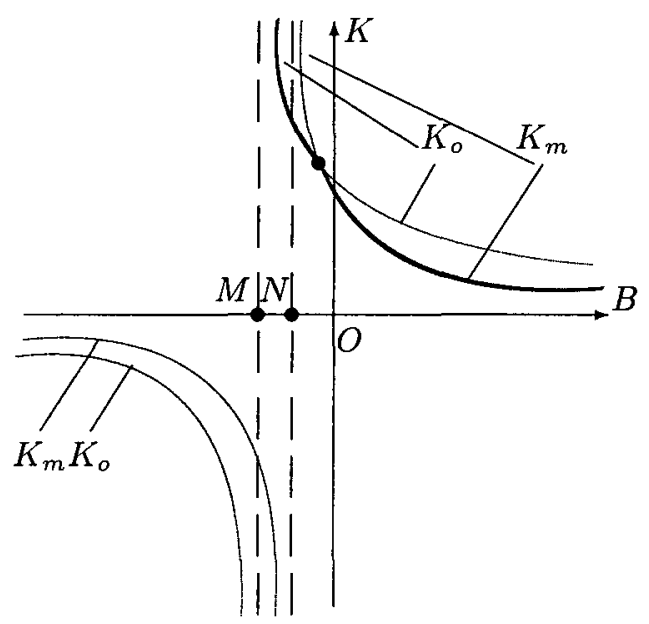

Fig. 2: QUALITATIVE PICTURE OF CRITICAL PERMEABILITIES VERSUS CONCENTRATION GRADIENT

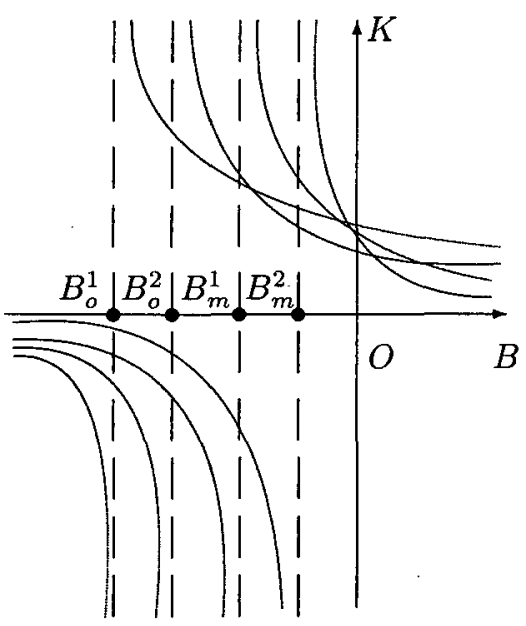

Fig. 3: CRITICAL PERMEABILITIES VERSUS CONCENTRATION GRADIENT FOR TWO-LAYERED SYSTEM types of perturbations are indicated by the subscripts. On this picture we illustrate the nontrivial event when mechanical equilibrium for the system heated from below can be unstable in the upper layer, while one can be stable in the lower layer. The possibility of instability is connected with evolution of oscillatory perturbations.

The threshold values of $B$ in the points $B_{m}^{1}, B_{m}^{2}$ are usually small as compared to reservoir values. The point corresponding to real reservoir conditions belongs, as a rule, to the region of absolute stability.

\section{CONVECTIVE STABILITY IN A THICK NATURAL RESERVOIR}

Consider application of the stability criteria derived under conditions of the Karachaganak Field. This oil-gas-condensate field is located in Precaspian Depression (Kazakhstan). Reservoir thickness is $1650 \mathrm{~m}$, including $1433 \mathrm{~m}$ of a gas cap. The reservoir is extremely heterogeneous and anisotropic. The reservoir fluids are heavy gascondensate and volatile oil. Diagnosis of stability for this field allows to validate the procedure of 1D compositional grading in order to predict depth distribution of reserves.

The multi-component reservoir mixture is modelled by two pseudo-components, of which the lighter corresponds to methane. The following values are selected as defining parameters [15]: $C^{0}=0.4 ; T^{0}=360 \mathrm{~K} ; p^{0}=60 \mathrm{MPa} ; C_{p}=3 \times$ $10^{3} \mathrm{~m}^{2} / \mathrm{s}^{2} \mathrm{~K} ; \quad \rho_{0}=650 \mathrm{~kg} / \mathrm{m}^{3} ; \lambda=2.5 \mathrm{~kg} \cdot \mathrm{m} / \mathrm{s}^{3} \mathrm{~K} ;$ $C_{m}^{j}=1.79 \times 10^{6} \mathrm{~m}^{2} / \mathrm{s}^{2} \mathrm{~K} ; \eta=10^{-4} \mathrm{~kg} / \mathrm{m} \mathrm{s} ; L=1433 \mathrm{~m} ;$ $A=0.03 \mathrm{~K} / \mathrm{m} ; B=-4 \times 10^{-5} \mathrm{~m}^{-1} ; \beta_{T}=10^{-4} \mathrm{~K}^{-1}$; $\beta_{C}=0.1, D=10^{-9} \mathrm{~m}^{2} / \mathrm{s}$.

The characteristics of stability are as follows: $\phi_{m}=5.8 \times 10^{-4}, \phi_{o}=10.22$. Thus, according to the criteria (45), (46), the stability is absolute, and the Karachaganak mixture is far from the region where an instability becomes possible. It is the multicomponent nature of the fluid that defines absolute stability of the equilibrium state: for homogeneous fluid the convection is always possible under some high value of the permeability $[3,7]$. Fundamental errors thus arise from neglect of the multicomponent nature of the fluid.

Similar calculations were made for the Tengiz field [15] $\left(C^{0}=0.36 ; T^{0}=430 \mathrm{~K} ; p^{0}=85 \mathrm{MPa} ; C_{p}=\right.$ $2.75 \times 10^{3} \mathrm{~m}^{2} / \mathrm{s}^{2} \mathrm{~K} ; \rho_{0}=620 \mathrm{~kg} / \mathrm{m}^{3} ; \lambda=1.7 \mathrm{~kg} \cdot \mathrm{m} / \mathrm{s}^{3} \mathrm{~K} ;$ $\eta=2 \times 10^{-4} \mathrm{~kg} / \mathrm{m} \mathrm{s} ; L=1500 \mathrm{~m} ; A=0.05 \mathrm{~K} / \mathrm{m}$; $\left.B=-2 \times 10^{-5} \mathrm{~m}^{-1}\right)$. In this case it was found that when the kinetic effects are taken into account $K_{m}=-3.25 \times 10^{-17} \mathrm{~m}^{2}$ and $K_{o}=-8.34 \times 10^{-16} \mathrm{~m}^{2}$, whereas without allowance for kinetic effects $K_{m}=$ $-5.27 \times 10^{-17} \mathrm{~m}^{2}$ and $K_{o}=-2.49 \times 10^{-17} \mathrm{~m}^{2}$. The homogeneous model gives a value of the vertical 
critical permeability equal to $3.43 \times 10^{-14} \mathrm{~m}^{2}$. Thus, in this case also the multicomponent nature of the mixture is an important factor, which must be taken into account if correct conclusions are to be drawn concerning the stability of the mixture.

Nevertheless, the oscillatory instability becomes possible at some extreme reservoir conditions. For example, value of the thermal expansion $\beta_{T}$ can be of order $10^{-3}$ for some gases and liquids [16]. In this case, and for other data from the example above, the value of the critical gradient $B_{o, c r}$ corresponding to the point $M$ on Fig.2 equals $-3.9 \times 10^{-5} \mathrm{~m}^{-1}$ which is close to the Karachaganak's gradient (the critical gradient is defined from (46)). The critical gradient becomes even higher than Karachaganak's one if we pose $C_{p}=3.3 \times 10^{3} \mathrm{~m}^{2} / \mathrm{s}^{2} \mathrm{~K}$. In this case $B_{o, c r}=-4,3 \times 10^{-5} \mathrm{~m}^{-1}$. The corresponding critical permeability with respect to oscillatory perturbations is equal to $2 \times 10^{-13} \mathrm{~m}^{2}$ which is about 20 times higher than the critical permeability for a homogeneous fluid: $K_{h o m}=1.2 \times 10^{-14} \mathrm{~m}^{2}$. For the value of $B$ equal to $0.5 B_{o, c r}$ the critical permeabilities calculated for the homogeneous fluid and for the binary mixture are of the same order: $K_{o}=2.4 \times 10^{-14} \mathrm{~m}^{2}$, $K_{\text {hom }}=1.2 \times 10^{-14} \mathrm{~m}^{2}$.

The monotonic instability becomes possible only for extremely small values $f_{\lambda} / f_{d}$, if the diffusion anisotropy much exceeds the heat conduction one. Additionally, values of the diffusion coefficient and the thermal expansion must be high. For the values of $f_{\lambda}=1, f_{d}=f_{k}=50, f_{\lambda} / f_{d}=0.02$, $\beta_{T}=10^{-3}, D=10^{-9} \mathrm{~m}^{2} / \mathrm{s}$, the value of critical gradient with respect to monotonic perturbations equals to $B_{m, c r}=-1.3 \times 10^{-4} \mathrm{~m}^{-1}$, which much exceeds the Karachaganak's gradient of the concentration. The critical permeability defined by (47), with $n=1, k^{2}=\pi^{2} /\left(f_{k} f_{d}\right)^{1 / 2}$, is equal to $1.98 \times 10^{-14} \mathrm{~m}^{2}$.

Thus, the instability with respect to oscillatory perturbations becomes possible for the fluids with high thermal expansion coefficients. The anisotropy with respect to monotonic perturbations is possible for anomalous anisotropy properties of a porous medium and high values of the diffusion coefficient. The values of critical permeabilities, if positive, are of order $10^{-14} \mathrm{~m}^{2}$, which is quite comparable with ordinary values of reservoir permeabilities.

To clarify the dependence of critical gradients and permeabilities on different fluid properties we have calculated these values in reservoir conditions for different model mixtures: gas $\left(95 \%\right.$ of $\mathrm{CH}_{4}$ and $5 \%$ of $\left.\mathrm{C}_{2} \mathrm{H}_{6}\right)$, gas-condensate $\left(70 \%\right.$ of $\mathrm{CH}_{4}$ and $30 \%$ of $\left.\mathrm{C}_{2} \mathrm{H}_{6}\right)$, light oil $\left(70 \%\right.$ of $\mathrm{CH}_{4}$ and $30 \%$ of $\left.\mathrm{C}_{7} \mathrm{H}_{16}\right)$, and heavy oil $\left(30 \%\right.$ of $\mathrm{CH}_{4}$ and $70 \%$ of $\left.\mathrm{C}_{7} \mathrm{H}_{16}\right)$. The properties of the mixtures have been chosen close to reservoir ones.

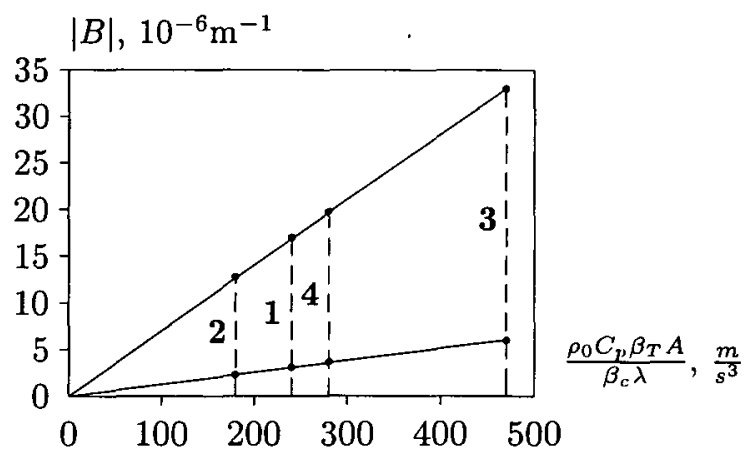

Fig. 4: ABSOLUTE VALUES OF THE CRITICAL GRADIENTS OF CONCENTRATION FOR DIFFERENT MIXTURES: 1 - gas, 2 gas-condensate, 3 - light oil, 4 - heavy oil.

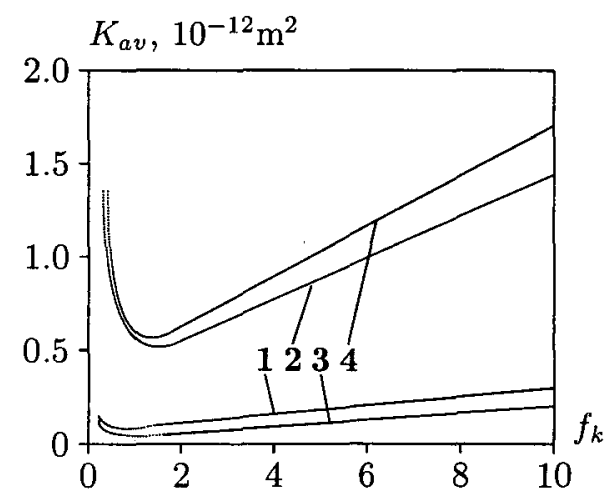

Fig. 5: DEPENDENCIES OF THE AVERAGE CRITICAL PERMEABILITY ON THE ANISOTROPY OF PERMEABILITY FOR DIFFERENT MIXTURES : 1 - gas, 2 - gascondensate, 3 - light oil, 4 - heavy oil.

Fig.4 shows the dependence of critical gradients $B_{m, c r}, B_{o, c r}$ on a common combined parameter (eqs. (52), (53)). The dependence of the average permeability $K_{a v}$ on the anisotropy parameter $f_{k}$ are shown on Fig.5. Therewith, the average permeability is defined for any layer as the mean square value of the two horizontal and one vertical permeabilities:

$$
K_{a v}^{j}=K_{0}^{j} \sqrt{\frac{1+2 f_{k}^{2}}{3}}
$$

One can note the nonmonotonic dependence of critical parameters on the "heaviness" of a fluid. This is explained by complex nature of dependence of these parameters on fluid properties.

As the anisotropy increases, the value of average critical permeability increases as well. Its asymptotic behavior is a straight line. The value of vertical critical permeability decreases with the 
anisotropy factor, tending to zero at infinity.

\section{CONCLUSIONS}

1. The problem of convective stability of a binary mixture in an anisotropic porous medium allows an exact analytical solution.

2. A segregated state of the multicomponent mixture is always more stable than that of the onecomponent one. It is the multicomponent nature of the mixture that defines the stability.

3. Under reservoir conditions the equilibrium is usually stable with respect to both monotonic and oscillatory perturbations. The equilibrium can become unstable with respect to the oscillatory perturbations for a mixture of high thermal conductivity and heat capacity.

4. The mechanical equilibrium for the system of some saturated porous layers heated from below can be unstable in the upper layer, while one can be stable in the lower layer. The possibility of instability is connected with evolution of oscillatory perturbations.

5. There exists critical (absolute) value of the lighter component concentration gradient above which the mixture is stable for any value of critical permeability. For concentration gradient below critical one, the critical permeability is defined as a threshold value above which the convection is observed.

6. If the critical permeability is negative, the equilibrium is stable for any reservoir permeability.

7. Characteristic values of critical permeabilities, if positive, are of order $10^{-13}-10^{-14} \mathrm{~m}^{2}$. These values are quite comparable with characteristic permeabilities of natural oil-gas-condensate reservoirs. Thus the convection is possible in high permeable zones of such reservoirs.

8. The anisotropy of a porous medium changes values of critical permeabilities, but it does not affect the boundaries of the regions of absolute stability with respect to monotonic and oscillatory perturbations.

9. The anisotropy correction factors for the values of critical permeabilities are the same for homogeneous fluid and for binary mixture.

10. As the anisotropy increases, the value of average critical permeability increases too, but the value of vertical critical permeability decreases.

\section{NOMENCLATURE}

$a, b, d \ldots .$. coefficients of the dispersion polynomial;

$B_{m, c r}, B_{o, c r}$ critical concentration gradients;

$C^{j} \ldots \ldots \ldots$ deviation of the concentration of the lighter component from a constant value $C_{0}$;

$C_{m}^{j} \ldots \ldots .$. total heat capacity of the porous medium and the mixture;

$C_{p} \ldots \ldots \ldots$ isobaric heat capacity of the mixture;

$D^{j}, D_{0}^{j} \ldots \ldots$ horizontal and vertical diffusion coefficients;

$\mathrm{D}_{\mathrm{t}}{ }^{j} \ldots \ldots \ldots$ dimensionless number of diffusive heat conduction;

$f_{d}^{j}, f_{k}^{j}, f_{\lambda^{j}} \ldots$ anisotropy factors for the diffusion coefficient, the permeability and the heat conductivity.

$\mathrm{G}^{j} \ldots \ldots \ldots$ gravity factor;

g......... gravity acceleration;

$K^{j}, K_{0}^{j} \ldots$ horizontal and vertical permeabilities;

$k_{i}(i=1,2), k$ wave numbers for the horizontal coordinates, $k^{2}=k_{1}^{2}+k_{2}^{2}$.

$L^{j} \ldots \ldots \ldots$ thickness of the layer of porous medium (or, of the natural reservoir).

$n \ldots \ldots \ldots$ wave number for the vertical coordinate;

$\mathrm{P}^{j} \ldots \ldots \ldots$ flow Prandtl number;

$\mathrm{P}_{\mathrm{d}}{ }^{j} \ldots \ldots \ldots$ diffusion-flow Prandtl number;

$p \ldots \ldots \ldots$ deviation of the pressure from the hydrostatic distribution;

$\mathrm{R}^{j} \ldots \ldots \ldots$ flow Rayleigh number;

$\mathrm{R}_{\mathrm{d}}{ }^{j}$....... diffusion-flow Rayleigh number;

$T^{j} \ldots \ldots \ldots$ deviation of the temperature from a constant value $T_{0}$

$\mathrm{T}_{\mathrm{d}}{ }^{j} \ldots \ldots \ldots$ dimensionless number of thermodiffusion;

$t \ldots \ldots \ldots$ time;

$\mathbf{u}^{j} \ldots \ldots \ldots$ velocity of the binary flow;

$w_{i} \ldots \ldots \ldots$ amplitude of perturbation of the i-th velocity component;

$x_{1}, x_{2} \ldots \ldots$ horizontal coordinates; 
$x_{3} \ldots \ldots \ldots$ vertical coordinate;

$\beta_{C}^{j}, \beta_{T}^{j} \ldots$ concentration and thermal expansion constants;

$\eta \ldots . \ldots$. viscosity;

$\lambda^{j}, \lambda_{0}^{j} \ldots \ldots$ horizontal and vertical heat conductivities;

$\mu^{j} \ldots \ldots \ldots$ effective chemical potential of the mixture;

$\kappa_{T}^{j} \ldots \ldots \ldots$ thermodiffusion coefficient;

$\rho \ldots \ldots \ldots$. density;

$\theta \ldots \ldots \ldots$ amplitude of the temperature perturbation;

$\xi \ldots \ldots \ldots$ amplitude of the concentration perturbation;

$\zeta \ldots \ldots \ldots$ amplitude of the pressure perturbation;

$\omega \ldots \ldots \ldots$ perturbation decrement;

$\Omega_{L}, \Omega_{K} \ldots$ scale and permeability factors.

\section{ACKNOWLEDGEMENTS}

The author is grateful to P. G. Bedrikovetsky (PETROBRAS, Brazil) and A. A. Shapiro (Engineering Research Center IVC - SEP, Denmark) for helpful discussions and valuable comments. Also I wish to thank V. D. Arnold (Moscow South-West High School) for the help in usage of $\mathrm{EATEX}_{\mathrm{E}}$.

\section{REFERENCES}

[1] Bott M.H.P., "The Interior of the Earth", Edward Arnold, London (1971).

[2] Nield D.A., "Onset of Thermoline Convection in a Porous Medium", Water Resources Research, Vol. 4, No. 3, pp. 553-559 (1968).

[3] Epherre J.R., "Critère d'Apparition de la Convection Naturelle dans une Couche Poreuse Anisotrope", Rev. Gen. Therm., Vol. 14, No. 168 , pp. 949-950 (1975).

[4] Sharma R.C., Kumari V. and Misra J.N., "Thermosolutal Convection in Compressible Fluids in a Porous Medium", J. Math. Phys. Sci., Vol. 24, No. 4, pp. 265-281 (1990).

[5] Poulicacos D. "The Effect of a Third Diffusing Component on the Onset of Convection in a Horizontal Porous Layer", Phys. Fluids, Vol. 28, pp. 3172-3174 (1985).
[6] Terrones G., Pearlstein A. "The Onset of Convection in a Multicomponent Porous Layer", Phys. Fluids, Vol. 1, pp.845-853 (1989).

[7] Bedrikovetsky P.G., Polonsky D.G. and Shapiro A.A., "Analysis of the Convective Instability of a Binary Mixture in a Porous Medium", Fluid Dynamics, No. 4, pp. 82-89, July (1993).

[8] Bedrikovetsky P.G., Polonsky D.G. and Shapiro A.A., "Convective Stability of a Binary Mixture in an Anisotropic Porous Medium", Entropie, No. $184 / 185$, pp. 73-80, (1994).

[9] Polonsky D.G. "Compressibility Effects under Conditions of Convective Stability of a Miscible Fluids in a Porous Medium", Fluid Dynamics, No. 1, pp. 11-17, January (1995).

[10] Barenblatt G.I., Entov V.M. and Ryzhik V.M., "Theory of Fluid Flows Through Natural Rocks", Kluwer Academic Publisher, Dordrecht, Boston, London (1991).

[11] Bedrikovetsky P.G., "Mathematical Theory of Oil and Gas Recovery", Kluwer Academic Publisher, Dordrecht, Boston, London (1993).

[12] Gershuni G.E. and Dzukhovitsky E.M., "Convective Stability of Incompressible Liquid", Nauka, Moscow (1972).

[13] Landau L.D. and Lifshitz E.M., "Fluid Mechanics (Course of Theoretical Physics, Vol. 6)", 2nd Ed., Pergamon Press, Oxford (1987).

[14] Gebhart B., Jaluria Y., Mahajan R. and Sammakia B., Buoyancy-Induced Flows and Transport, Hemisphere Publishing Corporation, Washington, New York, London (1988).

[15] Perepelichenko V.F., Recovery of components from Oil-Gas-Condensate Reservoirs, Nedra, Moscow (1990).

[16] Hirshfelder J.O., Curtiss Ch.F. and Bird R.B. Molecular Theory of Gases and Liquids, Wiley and Sons Inc., New York (1954). 